\title{
A Wedge-DCB Test Methodology to Characterise High Rate Mode-I Interlami- nar Fracture Properties of Fibre Composites
}

\author{
Sathiskumar A. Ponnusami ${ }^{1, *}$, Hao $\mathrm{Cui}^{1,2}$, Borja Erice $^{1}$, Mehtab V. Pathan ${ }^{1}$, and Nik Petrinic ${ }^{1}$ \\ ${ }^{1}$ Department of Engineering Science, University of Oxford, Oxford, United Kingdom. \\ ${ }^{2}$ School of Aerospace, Transport and Manufacturing, Cranfield University, Cranfield, United Kingdom.
}

\begin{abstract}
A combined numerical-experimental methodology is presented to measure dynamic Mode-I fracture properties of fiber reinforced composites. A modified wedge-DCB test using a Split-Hopkinson Bar technique along with cohesive zone modelling is utilised for this purpose. Three different comparison metrics, namely, strain-displacement response, crack propagation history and crack opening history are employed in order to extract unique values for the cohesive fracture properties of the delaminating interface. More importantly, the complexity of dealing with the frictional effects between the wedge and the DCB specimen is effectively circumvented by utilising right acquisition techniques combined with an inverse numerical modelling procedure. The proposed methodology is applied to extract the high rate interlaminar fracture properties of carbon fiber reinforced epoxy composites and it is further shown that a high level of confidence in the calibrated data can be established by adopting the proposed methodology.
\end{abstract}

\section{Introduction}

Delamination is known to be one of the crucial failure mechanisms in fibre reinforced composites, particularly for structures subjected to impact loads. Understanding this failure mechanism is important, especially for aerospace structures, where their presence can severely compromise the structural integrity. Designing a composite structure against such a failure mechanism requires the material property (i.e., fracture toughness) of the composite interfaces i.e., the layers between the plies susceptible to delamination. The properties of interest for designers include the fracture toughness under Mode-I, Mode-II and mixed-mode loading conditions. It is known that, in general, the Mode-I opening fracture toughness is lower than the toughness in Mode-II, hence it becomes a critical property assuming importance in the design process.

Characterisation of delamination fracture properties at high strain rates is still a standing issue due to the inherent challenges posed by the high strain rate experimental methodology. In particular, the test methodology of fracture tests at high loading rates is not well-established when compared with in-plane failure characterization of composites at high strain rates. Over the last decade, several experimental studies were conducted to measure the high rate interlaminar fracture toughness using different test approaches that include several DCB-based configurations, compact tension and compression, planar plate impact and edge notched specimens. A comprehensive review of different approaches for measuring rate-dependent Mode-I interlaminar toughness can be found in [1]. Some common drawbacks of several test methods are the iner-

\footnotetext{
*e-mail: sathis.ponnusami@eng.ox.ac.uk
}

tial effects and the unsymmetrical opening of the cracks at high loading rate which leads to a mixed-mode fracture condition instead of being purely Mode-I.

Among the different approaches, the DCB or its modified versions were the most commonly used test setup for the determination of rate-dependent fracture toughness of the interface. The standard DCB test configuration was successfully utilised to measure the interlaminar fracture toughness for low to moderate rates of loading using a screw-driven or a servo-hydraulic driven testing machine [2-6]. Drop tower-loaded DCB has also been employed for the test, whereby the DCB specimen is mounted so as to enable tearing of "free arms" on specimen in Mode-I by the vertically-dropping wedge. [7].

For the high rate testing, it is not feasible to use the standard DCB configuration because of technical difficulties in using the load application methodology, during which the inertial effects induce an unsymmetrical crack propagation. To overcome the above limitation, an alternative DCB test configuration has been proposed in the literature [1]. A wedge-loaded DCB in a Hopkinson Bar or drop tower impact set-up is found to be suitable for inducing a pure Mode-I crack. However, one of the drawbacks in wedge-DCB test is that the friction coefficient between the wedge and the DCB specimen needs to be determined in order to make use of the measured data i.e., the loaddisplacement response.

From the literature, it is understood that there is no clear consensus on the appropriate test methodology for measuring Mode-I interlaminar fracture toughness of fiber composites. It is therefore, the objective of the present research to propose a numerical-experimental methodology using a wedge-DCB test in a Split-Hopkinson Bar setup 
to determine the Mode-I delamination toughness. The difficulties associated with the wedge-loaded DCB test (i.e., the friction and inertial effects) are circumvented by using an inverse modelling approach along with a carefully planned data acquisition in the experiments.

\section{Experiments}

\subsection{Specimen details}

A carbon fiber-reinforced composite laminate of type IM7/8552 from Hexcel is utilised for the study. Unidirectional laminate panel of nominal thickness of $3 \mathrm{~mm}$ was manufactured along with a Teflon insert in the midplane of the laminate for precrack requirements. DCB specimens are cut to obtain the following dimensions: length of the beam $=120 \mathrm{~mm}$, width $=20 \mathrm{~mm}$ and thickness $=3$ $\mathrm{mm}$. A precrack of length $=32 \mathrm{~mm}$ is used.

\subsection{Test setup and data processing}

The test set-up involves a steel wedge being pushed through the DCB specimen at high loading rates by using a Split-Hopkinson bar set up as shown in the Fig. 1. For the sake of brevity, theoretical background and data reduction techniques of SHPB test is not included in this paper, readers can refer to [8] for further details. A strain gauge is mounted on the top surface of the DCB upper arm at the location corresponding to the initial crack tip. A Kirana ultra-high speed camera was used to capture images with a frame rate of 500,000 frames per second. A speckle pattern was applied to transverse DCB face for subsequent DIC analyses. Three parameters of interests are extracted through postprocessing of the experimental data: (a) strain history from the strain gauge mounted on the DCB top surface at the initial crack tip location, (b) crack length using the images recorded by the high-speed camera and (c) crack opening at the initial crack tip location by tracking two corresponding points using DIC image correlation.

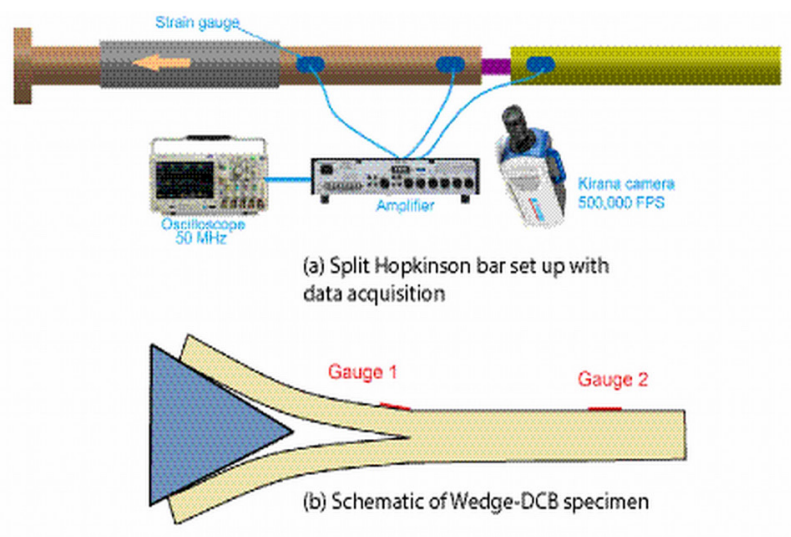

Fig. 1. Experimental setup of the wedge-DCB specimen in Split Hopkinson Pressure Bar system.

\section{Inverse modelling approach}

The present research adopts an inverse modelling approach employing finite element simulations involving cohesive elements to determine the interlaminar fracture toughness (energy) of the composite specimen. The following subsections provide details of the finite element model, material parameters and the approach to determine the cohesive law characterised by interlaminar cohesive strength and fracture energy.

\subsection{Finite element modelling}

A two-dimensional finite element model of the wedgeDCB test is set up using the finite element package Abaqus as shown in Fig. 2. The unidirectional composite beam arms of the DCB are modelled using quadrilateral plane strain elements with linear elastic and transversely isotropic model describing their constitutive behavior. The elastic properties of the lamina is taken from authors' work [9]. A layer of 2D cohesive elements of thickness $0.01 \mathrm{~mm}$ is inserted between the beam arms to simulate interface delamination. A bilinear traction-separation law characterized by three parameters, namely the cohesive stiffness, strength and fracture energy is utilised to model the delamination initiation and propagation. The finite element mesh is ensured to be sufficiently fine such that the number of cohesive elements is adequate in order to properly resolve the cohesive zone. The steel wedge is modelled as linear elastic with isotropic material properties, $E=210 \mathrm{GPa}$ and $v=0.3$. The mass densities of the steel and the composite are taken as 7700 and $1560 \mathrm{~kg} / \mathrm{m}^{3}$ respectively. The loading conditions of the wedge DCB setup in the Split Hopkinson bar system is simulated through applied boundary conditions in the FE model as shown in the figure. The simulations were conducted using Abaqus explicit solver.

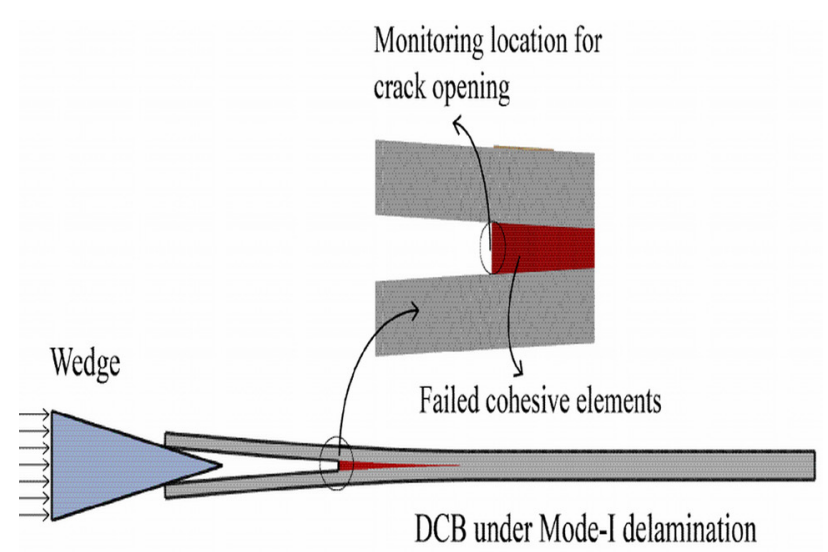

Fig. 2. Finite element model of the WDCB specimen with a layer of cohesive elements.

\subsection{Comparison metrics}

As discussed in the previous sections, a major challenge in processing and interpreting the data obtained in the wedgedriven DCB test lies in modelling the frictional effects between the wedge and the DCB surfaces. The objective 
of this work is to eliminate this complexity by introducing an alternate form of the load-displacement curve and two other response metrics for the comparison in the inverse modelling procedure. Firstly, instead of a conventional load-displacement response, an alternate measure i.e. strain measured on the top surface of DCB using a strain gauge and applied wedge displacement is used. The bending strain measured at the location in the DCB arm corresponding to the initial crack tip (see Fig. 1) can be used directly to correlate with the load applied at the wedge-DCB contact point through beam theory, if necessary. The other two metrics are the crack length $v s$ wedge displacement and the crack opening (at initial crack tip location) $v s$ the wedge displacement histories. The objective of the inverse modelling procedure is to determine the interface cohesive parameters with which the simulation results match well with the experimental data in terms of the three metrics described above. Among the three cohesive parameters, the cohesive stiffness parameter is set to a sufficiently high value to avoid any artificial compliance without inducing any numerical issues. Thus, the procedure aims to determine two other cohesive parameters, the cohesive strength and the energy. To this end, the following procedure is adopted: (i) guess values for the cohesive strength and the energy are chosen and the simulation is conducted, (ii) the resulting three metrics are then compared with the experimental data and (iii) the process is repeated until the metrics obtained from the simulations matches with the experimental results. The corresponding interface properties are then established as the interface cohesive strength and fracture energy of the composite interface.

\section{Results and discussion}

The inverse modelling procedure discussed above is followed to conduct a series of simulations to identify the best match for the interface properties by using the three metrics. Through numerous iterative simulations, the converged interface properties are obtained. In order to highlight the procedure and explain the effect of the cohesive strength and the fracture energy, the results of the simulations corresponding to three chosen values for each interface parameter (i.e., the cohesive strength and the fracture energy) are discussed in the following sections. In the experiments, two tests were conducted and the results are plotted in terms of an average (dotted line) with the scatter in experiments shown as gray shaded region.

\subsection{Strain-displacement response}

The results of the simulations in terms of the straindisplacement response are summarised in Figs. 3 and 4, showing the effect of cohesive strength and the fracture energy on the response respectively.

Regarding the effect of cohesive strength, several values of the cohesive strength, $\sigma_{\mathrm{c}}$ ranging from $25 \mathrm{MPa}$ to $150 \mathrm{MPa}$ with increments of $25 \mathrm{MPa}$ were considered

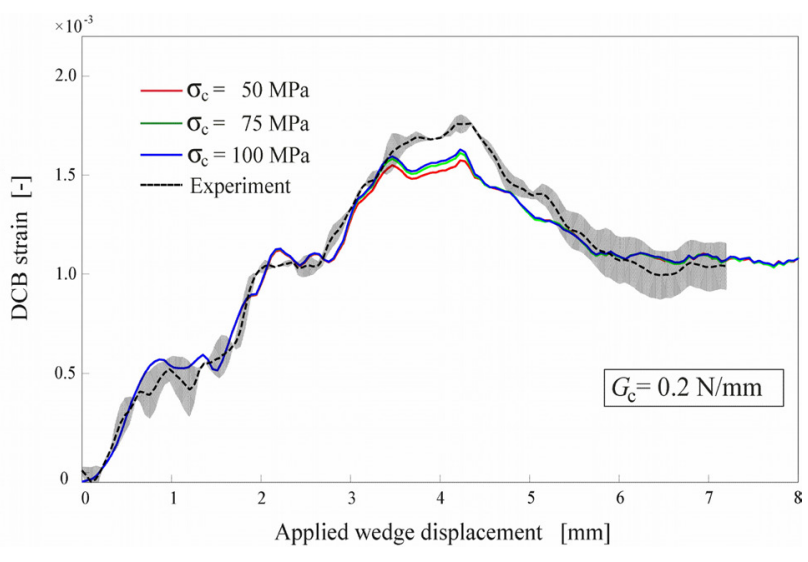

Fig. 3. Effect of strength on strain-displacement response.

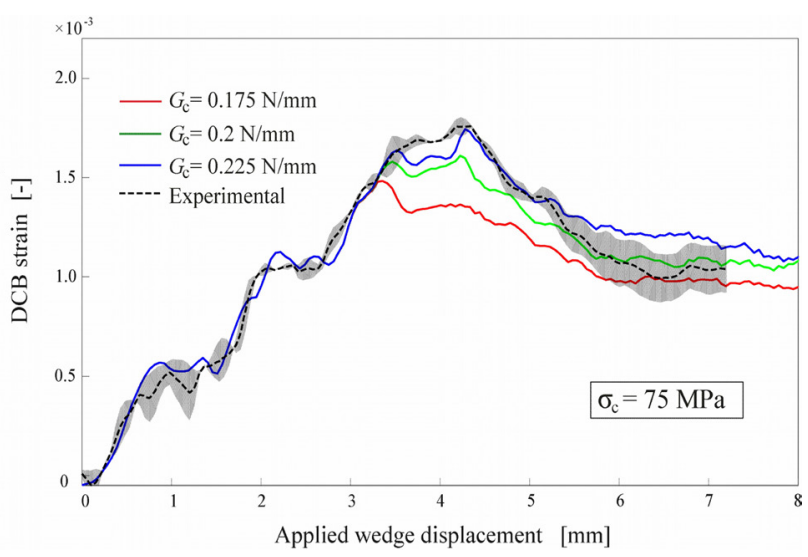

Fig. 4. Effect of fracture energy on strain-displacement response.

for simulations. The strain-displacement response corresponding to three selected values of cohesive strength, $\sigma_{\mathrm{c}}=$ 50,75 and $100 \mathrm{MPa}$ is plotted in Fig. 3. The fracture energy for the simulations is taken as $G_{\mathrm{c}}=0.2 \mathrm{~N} / \mathrm{mm}$. It is observed that the cohesive strength has a minor influence on the response. This is expected as the cohesive strength becomes a numerical parameter at structural scale, particularly when the fracture process zone length is smaller compared to the specimen scale. However, the cohesive strength cannot be assigned very small or very large values as the former would introduce artificially large process zone, while the latter would induce numerical issues (a very fine mesh would be necessary in such case). From the results reported in the figure for three different values of the cohesive strength $\sigma_{\mathrm{c}}$, a value of $75 \mathrm{MPa}$ is chosen as the cohesive strength of the interface as it matches well with the experimental response and any further increase in the cohesive strength does not alter the response noticeably.

To show the effect of fracture energy, the responses corresponding to three chosen values given by $G_{\mathrm{c}}=0.175$, 0.2 and $0.225 \mathrm{~N} / \mathrm{mm}$ are shown in Fig. 4. The cohesive strength of the interface elements is fixed as $\sigma_{\mathrm{c}}=75 \mathrm{MPa}$. Unlike cohesive strength, the fracture energy strongly influences the response as observed from the Fig.4. From the 
results, it can be observed that the response corresponding to $G_{\mathrm{c}}=0.2 \mathrm{~N} / \mathrm{mm}$ matches very well with the experiment.

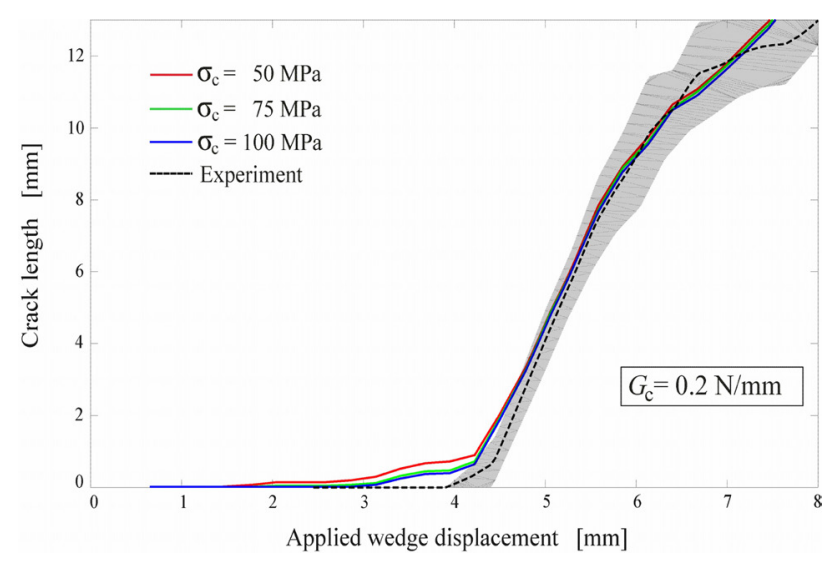

Fig. 5. Effect of cohesive strength on crack length history.

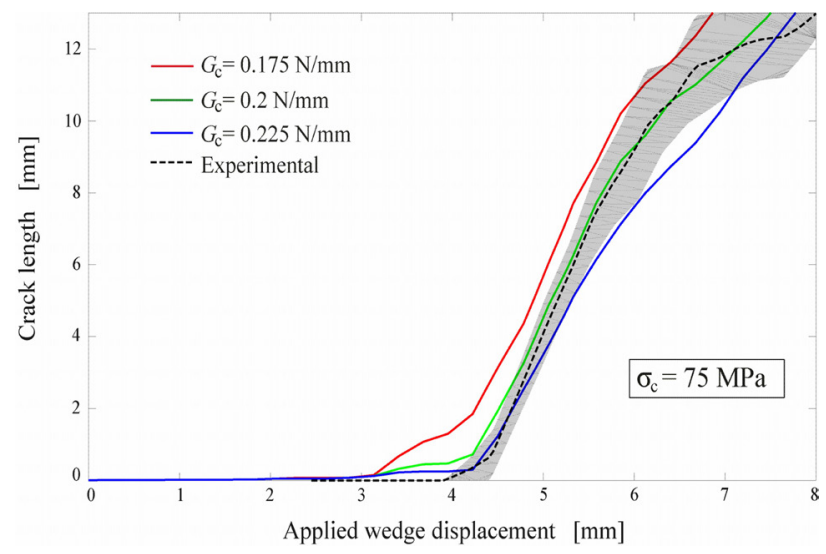

Fig. 6. Effect of fracture energy on crack length history.

\subsection{Crack length-displacement history}

The second metric, namely the crack length is determined from the simulations by using the number of failed cohesive elements in the interface. The results are plotted in the Figs. 5 and 6 for the chosen values of the cohesive strength and energy. From this plot and the straindisplacement response in the Figs. 3 and 4, one can observe that the crack initiation occurs approximately when the strain ("load") reaches its peak, following which the crack length increases monotonically with further loading. It is evident from the plot that the numerical and the experimentally observed crack length histories are in good agreement for the value of cohesive strength, $\sigma_{\mathrm{c}}=75 \mathrm{MPa}$ and fracture energy, $G_{\mathrm{c}}=0.2 \mathrm{~N} / \mathrm{mm}$. The rate of increase in crack length is found to be approximately constant, thus indicating a steady delamination crack growth. Moreover, the calculated crack propagation velocity of $35 \mathrm{~m} / \mathrm{s}$ obtained from simulations is in good agreement with experiments.

\subsection{Crack opening-displacement history}

Crack opening displacement history is the third parameter that is considered as a comparison metric. From both the experiments and the simulations, crack opening is monitored at the location corresponding to the initial crack tip. The results of the crack opening is plotted in Figs.7 and 8 for the chosen values of the cohesive parameters. The opening at the initial crack tip remains zero until the crack starts propagating and the strain reaches the peak, as seen from strain and crack length histories. It can be observed again that the values of the cohesive strength and energy equal to $75 \mathrm{MPa}$ and $0.2 \mathrm{~N} / \mathrm{mm}$ is matching well with the experimentally observed crack opening history.

From the results, the crack opening rate can be calculated (slope of crack opening time history) and is found to be approximately equal to $0.25 \mathrm{~m} / \mathrm{s}$ with a good match between the experiments and the simulation is obtained. It is worth mentioning that the above-determined crack opening rate and the crack propagation velocity can serve as the rate parameter for rate-dependent fracture mechanics models, similar to the parameter 'strain rate' in rate-dependent continuum damage mechanics models.

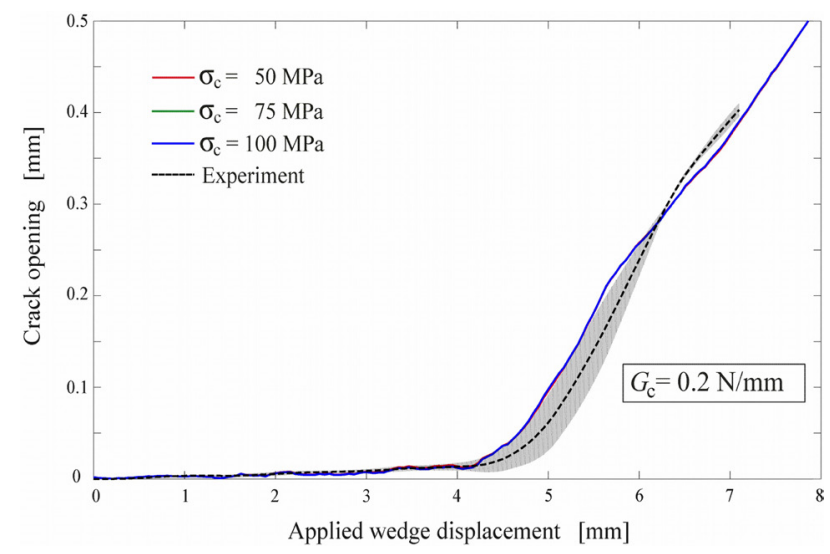

Fig. 7. Effect of cohesive strength on crack opening history.

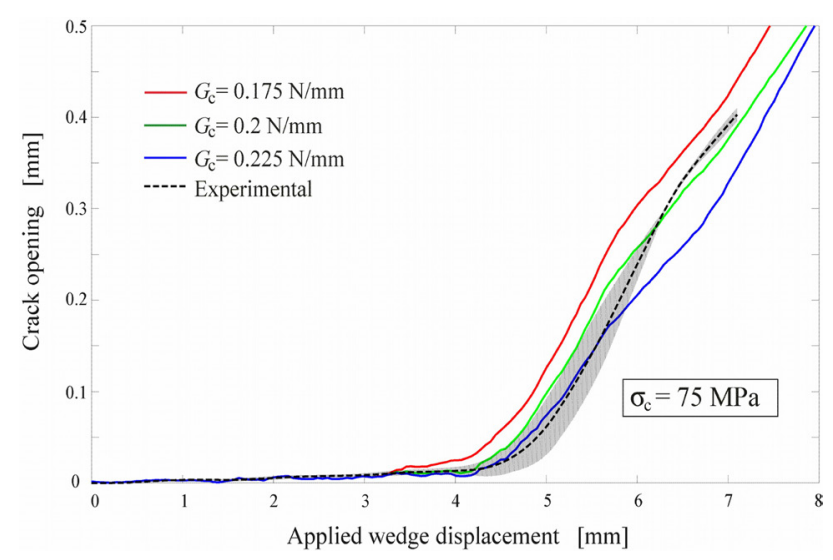

Fig. 8. Effect of fracture energy on crack opening history.

Upon considering the three metrics and their comparison between the simulated values and the experimentally 
measured values, it can be concluded that the dynamic delamination behavior of IM7/8552 composite material can be characterised by a bilinear cohesive law with its cohesive strength, $\sigma_{\mathrm{c}}=75 \mathrm{MPa}$ and delamination fracture energy, $G_{\mathrm{c}}=0.2 \mathrm{~N} / \mathrm{mm}$.

\section{Conclusions}

A methodology to determine dynamic delamination properties of fiber reinforced composites is proposed using an experimental-numerical approach. A three metric-based inverse modelling approach is adopted to quantify the interface material parameters using cohesive elements. Importantly, it has been shown that reliable values for the interface properties of the composite can be obtained without taking into account of the frictional effects between the wedge and the DCB arms. It is to be noted that the cohesive strength has a minimal influence on all the three metrics and it becomes a numerical parameter that can be assigned a value over a range in which it does not alter the response significantly. From the results, the dynamic fracture energy of the IM7/8552 composite interface is determined as $0.2 \mathrm{~N} / \mathrm{mm}$ with a crack opening rate and crack propagation velocity approximately given by $0.25 \mathrm{~m} / \mathrm{s}$ and $35 \mathrm{~m} / \mathrm{s}$ respectively.

\section{References}

1. M. May, Composites Part A: Applied Science and Manufacturing 81, 1 (2016)

2. A. Smiley, R. Pipes, Journal of composite materials 21, 670 (1987)

3. S. Mall, G. Law, M. Katouzian, Journal of composite materials 21, 569 (1987)

4. J.W. Gillespie, L.A. Carlsson, A.J. Smiley, Composites Science and Technology 28, 1 (1987)

5. R. Fracasso, M. Rink, A. Pavan, R. Frassine, Composites science and technology 61, 57 (2001)

6. H. Zabala, L. Aretxabaleta, G. Castillo, J. Aurrekoetxea, Composite Structures 121, 75 (2015)

7. M.C. de Verdiere, A. Skordos, M. May, A. Walton, Engineering Fracture Mechanics 96, 11 (2012)

8. H. Cui, M. Yasaee, S.R. Hallett, I.K. Partridge, G. Allegri, N. Petrinic, Composites Part A: Applied Science and Manufacturing 106, 24 (2018)

9. D.M. Thomson, B. Erice, H. Cui, J. Hoffmann, J. Wiegand, N. Petrinic, Composite Structures 184, 299 (2018) 
